\title{
The Inverse Problem of the Calculus of Variation
}

\author{
Estomih Shedrack Massawe \\ Department of Mathematics, College of Natural and Applied Sciences, University of Dar es Salaam, Dar es Salaam, Tanzania
}

Email address:

emassawe2@gmail.com, estomihmassawe@yahoo.com

\section{To cite this article:}

Estomih Shedrack Massawe. The Inverse Problem of the Calculus of Variation. Science Journal of Applied Mathematics and Statistics. Vol. 4, No. 2, 2016, pp. 48-51. doi: 10.11648/j.sjams.20160402.15

Received: February 8, 2016; Accepted: March 9, 2016; Published: March 28, 2016

\begin{abstract}
In this paper, it is intended to determine the necessary and sufficient conditions for the existence and hence the construction of a Lagrangian $L(t, q, \dot{q})$ of a dynamical system from its equations of motion. The existence of a Lagrangian is vital importance for the Hamiltonian description of a dynamical system since via the Legendre transformation $L \rightarrow H=\sum_{i=1}^{n} p_{i} \dot{q}_{i}-L(t, q, \dot{q})$ we get the Hamiltonian of the system [1,2]. It is also intended to show that the solution of the realization problem for the Hamiltonian system reduces to solving an inverse problem.
\end{abstract}

Keywords: Inverse Problems, Calculus of Variation, Realization Problem, Hamiltonian Systems

\section{Introduction}

In practice it often happens that the mathematical description of a system in terms of state variables is very complex or even not known but the external variables can be determined from experimental measurements or other considerations [3]. This difficulty is one reason for the frequent use of a "black-box" description. However it is very useful to find a system in state space form to which the set of the external variables correspond. In "Realization problem" we start with the external behaviour of a system and attempt to obtain the state space description. This idea is very necessary in control theory.

The Hamiltonian realization problem is described with a view of the inverse problem in classical being its special case; that is if the inverse problem can be solved, then there is a special case of the Hamiltonian realization problem that is solved. In realization problem for nonlinear input-output system $(U, Y, S)$ with $U$ the input space, $Y$ the output space and $S$ the system is to find a manifold $M$ called the state space with initial conditions $x(0)=x_{0}$ and functions $f: M \times U \rightarrow T M, h: M \rightarrow \mathbb{R}^{r}$ such that

$$
\left\{\begin{array}{l}
\dot{x}=f(x, u), \\
y=h(x),
\end{array} \quad x(0)=x_{0} ; x \in M\right.
$$

realizes the input-output system $(U, Y, S) . T M$ is the tangent bundle of $M$ [4].

\section{Formulation of the Inverse Problem}

The formulation of the inverse problem is as follows: Consider the Lagrange's equation

$$
L_{k}(q)=\frac{d}{d t} \frac{\partial L}{\partial \dot{q}^{k}}(t, q, \dot{q})-\frac{\partial L}{\partial q^{k}}(t, q, \dot{q})=0, k=1, \ldots, n .
$$

Consider also a holonomic Newtonian system

$$
\ddot{q}^{k}-f^{k}(t, q, \dot{q})=0 ; f^{k} \in C^{2}\left(\mathbb{R}^{2 n+1}\right), k=1, \ldots, n,
$$

or equivalently in fundamental form [3].

$$
\begin{gathered}
F_{k}=A_{k i}(t, q, \dot{q}) \ddot{q}^{i}+B_{k}(t, q, \dot{q})=0 \\
A_{k i} \in C^{2}\left(\mathbb{R}^{2 n+1}\right),\left|A_{i j}\right|\left(\mathbb{R}^{2 n+1}\right) \neq 0, i, k=1, \ldots, n .
\end{gathered}
$$

The inverse problem then consists of studying the 
conditions under which there exists Lagrangin $L(t, q, \dot{q})$ such that equations (2) coincides with equations (4) i.e.

$$
\frac{d}{d t} \frac{\partial L}{\partial \dot{q}^{k}}-\frac{\partial L}{\partial q^{k}}=A_{k i}(t, q, \dot{q}) \ddot{q}^{i}+B_{k}(t, q, \dot{q}), i, k=1, \ldots, n .
$$

Expansion of equation (2) yields

$$
\frac{\partial^{2} L}{\partial \dot{q}^{k} \partial \dot{q}^{i}} \ddot{q}^{i}+\frac{\partial^{2} L}{\partial \dot{q}^{k} \partial q^{i}} \dot{q}^{i}+\frac{\partial^{2} L}{\partial \dot{q}^{k} \partial t}-\frac{\partial L}{\partial q^{q}}=0, i, k=1, \ldots, n .
$$

Equation (5) then demands the validity of the equations

$$
\begin{gathered}
\frac{\partial^{2} L}{\partial \dot{q}^{k} \partial \dot{q}^{i}}=A_{k i}, \\
\frac{\partial^{2} L}{\partial \dot{q}^{k} \partial \dot{q}^{i}} \ddot{q}^{i}+\frac{\partial^{2} L}{\partial \dot{q}^{k} \partial t}-\frac{\partial L}{\partial q^{q}}=B_{k}, \quad i, k=1, \ldots, n .
\end{gathered}
$$

The following definition is necessary for the statement of existence of the Lagrangian.

\section{Definition}

The Lagrangian $L(t, q, \dot{q})$ is called regular/degenerate in a region $\mathbb{R}^{2 n+1}$ of points $(t, q, \dot{q})$ when the Hessian determinant $|H|\left(\mathbb{R}^{2 n+1}\right)=\left|\frac{\partial^{2} L}{\partial \dot{q}^{2} \partial \dot{q}^{j}}\right|\left(\mathbb{R}^{2 n+1}\right), j=1, \ldots, n \quad$ is non-null/null in it with the possible exception of a (finite) number of isolated zeros, [5]

The solution of the inverse problem needs the following ingredients:

Consider a system of $n$ second order ordinary differential equations

$$
F_{i}(q)=F_{i}(t, q, \dot{q}, \ddot{q})=0, i=1, \ldots, n .
$$

Define the variations of admissible one-parameter paths $P=\left\{q^{k}(t, \delta)\right\}, \quad t \in\left(t_{1}, t_{2}\right), \delta \in O_{\varepsilon}^{13}, k=1, \ldots, n$ which are at least $C^{2}$ in $t$ and $C^{1}$ in $\delta$ but not necessarily solutions of $F_{i}(q)=0$ by

$$
\begin{aligned}
& N^{k}(t)=\left.\frac{\partial q^{k}}{\partial \delta}\right|_{\delta=0}, \dot{N}^{k}(t)=\left.\frac{\partial \dot{q}^{k}}{\partial \delta}\right|_{\delta=0}, \\
& \ddot{N}_{k}(t)=\left.\frac{\partial \ddot{q}^{k}}{\partial \delta}\right|_{\delta=0}, k=1, \ldots, n .
\end{aligned}
$$

The variational forms of $F_{i}$ are given by

$$
M_{i}(N)=a_{i k}(t) N^{k}+b_{i k}(t) \dot{N}^{k}+c_{i k} \ddot{N}^{k}
$$

where $a_{i k}=\left.\frac{\partial F_{i}}{\partial q^{k}}\right|_{\delta=0}, \quad b_{i k}=\left.\frac{\partial F_{i}}{\partial \dot{q}^{k}}\right|_{\delta=0}, \quad c_{i k}=\left.\frac{\partial F_{i}}{\partial \ddot{q}^{k}}\right|_{\delta=0}, i, k=1, \ldots, n$.

\section{Definition}

A system of variational forms $\tilde{M}(N)$ is called the adjoint system of $M_{i}(N)$ defined by equations (11) if there exists a function $Q(N, \tilde{N})$ such that the Lagrange identity

$$
\tilde{N}^{i} M_{i}(N)-N^{i} \tilde{M}_{i}(\tilde{N})=\frac{d}{d t} Q(N, \tilde{N}), i=1, \ldots, n
$$

holds for all admissible variations [6].

[3] has shown that the possible structures of $M_{i}(N)$ and $Q(N, \tilde{N})$ are

$$
\begin{gathered}
\text { (a) } \tilde{M}_{i}(\tilde{N})=\tilde{N}^{k} a_{k i}-\frac{d}{d t}\left(\tilde{N}^{k} b_{k i}\right)+\frac{d^{2}}{d t^{2}}\left(\tilde{N}^{k} c_{k i}\right), \\
\text { (b) } Q(N, \tilde{N})=\tilde{N}^{i} b_{i j} N^{j}+\tilde{N}^{i} c_{i j} N^{j}-N^{j} \frac{d}{d t}\left(N c_{i j}\right), \\
i, j, k=1, \ldots, n .
\end{gathered}
$$

If $M_{i}(N)$ coincides with its adjoint $\tilde{M}_{i}(N)$ i.e. if $M_{i}(N)=\tilde{M}_{i}(N), i=1, \ldots, n, N=C^{2}$, then $M_{i}(N)$ is called self-adjoint.

By comparing equations (11) and (13) we get the conditions of self-adjointness for the variational forms:

$$
\begin{aligned}
& c_{i k}=c_{k i} \\
& b_{i k}+b_{k i}=2 \dot{c}_{k i} \quad, i, k=1, \ldots, n \\
& a_{i k}-a_{k i}=\ddot{c}_{k i}-\dot{b}_{k i}
\end{aligned}
$$

A system of ordinary differential equations is self-adjoint when its variational forms are self-adjoint.

[7] has further shown that

(A) A necessary and sufficient condition for a holonomic one-dimensional Newtonian system in the fundamental form $A(t, q, \dot{q}) \ddot{q}+B(t, q, \dot{q})=0 ; A, B \in C^{m}\left(\mathbb{R}^{3}\right), \quad m \geq 1$, $A\left(\mathbb{R}^{3}\right) \neq 0$ to be self-adjoint in a region $\mathbb{R}^{3}$ of points $(t, q, \dot{q})$ is that the condition

$$
\frac{\partial B}{\partial \dot{q}}=\frac{\partial A}{\partial t}+\frac{\partial A}{\partial q} \dot{q}
$$

Holds everywhere in $\mathbb{R}^{3}$.

(B) A necessary and sufficient condition for a holomic Newtonian $\quad$ system $\quad A_{k i}(t, q, \dot{q}) \ddot{q}+B_{k}(t, q, \dot{q})=0 \quad$, $i, k=1, \ldots, n$ satisfying the continuity and regularity conditions $A_{k i}, B_{k} \in C^{m}\left(\mathbb{R}^{2 n+1}\right), m \geq 2,\left|A_{k i}\right|\left(\mathbb{R}^{2 n+1}\right) \neq 0$, in a region $\mathbb{R}^{2 n+1}$ of points $(t, q, \dot{q})$ to be self-adjoint in 
$\mathbb{R}^{2 n+1}$, is that all of the following conditions are satisfied everywhere in $\mathbb{R}^{2 n+1}$ :

$$
\left\{\begin{array}{l}
A_{i j}=A_{j i}, \\
\frac{\partial A_{i k}}{\partial \dot{q}^{j}}=\frac{\partial A_{j i}}{\partial \dot{q}^{i}}, \\
\frac{\partial B_{i}}{\partial \dot{q}^{j}}+\frac{\partial B_{j}}{\partial \dot{q}^{i}}=2\left\{\frac{\partial}{\partial t}+\dot{q}^{k} \frac{\partial}{\partial q^{k}}\right\} A_{i j}, \\
\frac{\partial B_{i}}{\partial q^{j}}-\frac{\partial B_{j}}{\partial q^{i}}=\frac{1}{2}\left\{\frac{\partial}{\partial t}+\dot{q}^{k} \frac{\partial}{\partial q^{k}}\right\}\left(\frac{\partial B_{i}}{\partial \dot{q}^{j}}-\frac{\partial B_{j}}{\partial \dot{q}^{i}}\right),
\end{array} \quad i, j, k=1, \ldots, n\right.
$$

\section{Realization of Hamiltonian Systems}

In practice, it often happens that the mathematical description of system in terms of state variables is very complex or even not known but the external variables can be determined from experimental measurements or other considerations. This difficulty is one reason for the frequent use of "black-box" description. However it is very useful to find s system in state space form to which the set of the external variables correspond. In "Realization problem", we start with the external behaviour of a system of a system and attempt to obtain the state space description. The idea is very necessary in control theory.

We shall now establish the necessary conditions on the external behaviour of a Hamiltonian system such that we can construct a Hamiltonian system which generates this external behaviour. Let $\bar{w}=(\bar{y}, \bar{u})$ and $(\bar{y}, \bar{u}, \bar{x}): \mathbb{R} \rightarrow T^{*} Y \times M$ be a trajectory in $\sum_{i} \subset \sum(M, W, B, f)$. The variational principle assumes the existence of a parameter $\varepsilon \in \mathbb{R}$ such that a family of functions $(w(t, \varepsilon), x(t, \varepsilon))$ exists such that

i. $(w(., \varepsilon), x(., \varepsilon)) \in \sum_{i} \quad \forall \varepsilon$.

ii. $(w(., \varepsilon), x(., \varepsilon))$ is at least $C^{1}$ in $\varepsilon$ and in $t$.

iii. $(w(., 0), x(., 0))=(\bar{w}, \bar{x})$.

Then the variation of $(\bar{x}, \bar{w})$ is given by

$$
(\delta w(t), \delta x(t))=\left(\left.\frac{\partial w}{\partial \varepsilon}(t, \varepsilon)\right|_{\varepsilon=0},\left.\frac{\partial x}{\partial \varepsilon}(t, \varepsilon)\right|_{\varepsilon=0}\right) .
$$

Then

$$
\forall t,(\delta w(t), \delta(t)) \in T_{\bar{w}(t)} W \times T_{\bar{x}(t)} M .
$$

It can be shown

$\frac{d}{d t} \omega_{x(t)}\left(\delta_{1} x(t), \delta_{2} x(t)\right)=\omega_{w(t)}^{e}\left(\delta_{1} w(t), \delta_{2} w(t)\right)[8]$.

Consequently,

$$
\begin{aligned}
& \int_{t_{1}}^{t_{2}} \omega_{w(t)}^{e}\left(\delta_{1} w(t), \delta_{2} w(t)\right) d t \\
& =\omega_{x\left(t_{2}\right)}\left(\delta_{1} x\left(t_{2}\right), \delta_{2} x\left(t_{2}\right)\right)-\omega_{x\left(t_{1}\right)}\left(\delta_{1} x\left(t_{1}\right), \delta_{2} x\left(t_{1}\right)\right)
\end{aligned}
$$

$$
\begin{aligned}
& \forall(w(.), x(.)) \in \sum_{i} \text { and } \forall(\delta w(.), \delta x(.)) \text { of }(w(.), x(.)) . \\
& \text { If }(\delta w(.), \delta x(.)) \text { has compact support in }\left(t_{1}, t_{2}\right) \text { then }
\end{aligned}
$$

$$
\int_{t_{1}}^{t_{2}} \omega_{w(t)}^{e}\left(\delta_{1} w(t), \delta_{2} w(t)\right) d t=0 .
$$

This is the general variational principle and it involves only the external behaviour of the system. This formulation has a useful consequence for the Hamiltonian realization problem which can be seen in the following way.

Let $\left(W, \omega^{e}\right)$ denote a symplectic manifold and $Z$ denote a space of $C^{\infty}$ function $w: \mathbb{R} \rightarrow W$.We define a weak symplectic form $\Omega$ on $Z$ by

$$
\Omega_{w}\left(\delta_{1} w, \delta_{2} w\right)=\int_{-\infty}^{+\infty} \omega_{w(t)}^{e}\left(\delta_{1} w(t), \delta_{2} w(t)\right) d t
$$

where $w \in Z$ and $\delta_{1} w, \delta_{2} w$ are variations of $w$ with respect to $Z$ with compact support. If $\sum_{e}$ is an external system on $\left(W, \omega^{e}\right)$ such that $\sum_{e}$ is a submanifold of $Z$, then $\sum_{e}$ can be realized by a Hamiltonian system $\sum(M, W, B, f)$ iff $\sum_{e}$ is a Lagrangian submanifold of [9].

One procedure for realizing a Hamiltonian system is through solving an inverse problem of the calculus of variations as described below.

Consider a system of second order differential equations $f_{i}(q, \dot{q}, \ddot{q})=0, i=1, \ldots, m$. If we assume that the square matrix $\frac{\partial f_{i}}{\partial \ddot{q}_{j}}, i, j=1, \ldots, m$ has $\operatorname{det} \neq 0$, then the inverse problem is to find the conditions under which there exists the Lagrangian $L$ such that

$$
\frac{d}{d t}\left(\frac{\partial L}{\partial \dot{q}_{i}}\right)-\frac{\partial L}{\partial q_{i}}=f_{i}(q, \dot{q}, \ddot{q}), i=1, \ldots, m .
$$

We solve this problem through variation methods. Consider a family of curves in $\mathbb{R}^{m}$ given by $q(t, \varepsilon)$. The variations $\delta q_{i}, i=1, \ldots, m$ are defined by

$$
\delta q_{k}(t)=\left.\frac{\partial q_{k}}{\partial \varepsilon}(t, \varepsilon)\right|_{\varepsilon=0}, k=1, \ldots, m .
$$

Also the variational form are defined by

$$
M_{i}(\delta q)=\left.\left(\sum_{k=1}^{m} \frac{\partial f_{i}}{\partial q_{k}} \delta q_{k}+\frac{\partial f_{i}}{\partial \dot{q}_{k}} \delta \dot{q}_{k}+\frac{\partial f_{i}}{\partial \ddot{q}_{k}} \delta \ddot{q}_{k}\right)\right|_{\varepsilon=0}
$$

where

$$
f_{i}=f_{i}(q(t, \varepsilon), \dot{q}(t, \varepsilon), \ddot{q}(t, \varepsilon))
$$


Assume that there exists other variational forms $\tilde{M}_{1}(\tilde{\delta})$ such that we can define a function $Q$ where

$$
\sum_{i=1}^{m}\left(\tilde{\delta} q_{i} M_{i}(\delta q)-\delta q_{i} \tilde{M}_{i}(\tilde{\delta} q)\right)=\frac{d}{d t} Q(\delta q, \tilde{\delta} q)
$$

$\forall \delta q$ and $\tilde{\delta} q$ of $q(., \varepsilon)$ defined by equations (22).

If $\tilde{M}_{i}=M_{i}$, then there exists locally a function $L(q, \dot{q})$ such that

$$
\frac{d}{d t}\left(\frac{\partial L}{\partial \dot{q}_{i}}\right)-\frac{\partial L}{\partial q_{i}}=f_{i}
$$

Instead of taking $f_{i}(q, \dot{q}, \ddot{q})=0$ now we take $f_{i}(q, \dot{q}, \ddot{q})=u_{i}, i=1, \ldots, m$. If the inverse problem of calculus of variation for $f_{i}$ has a solution for the Lagrangian $L$, then the realization of the system $f_{i}(q, \dot{q}, \ddot{q})=u_{i}$, $i=1, \ldots, m$ is

$$
\begin{cases}\frac{d}{d t}\left(\frac{\partial L}{\partial \dot{q}_{i}}\right)-\frac{\partial L}{\partial q_{i}}, & i=1, \ldots, m \\ y_{i}=q_{i}, & i=1, \ldots, m .\end{cases}
$$

$\operatorname{Det}\left(\frac{\partial f_{i}}{\partial \ddot{q}_{i}}\right) \neq 0$ implies that $\operatorname{det}\left(\frac{\partial^{2} L}{\partial \dot{q}_{i} \partial \dot{q}_{j}}\right) \neq 0$.

If we define $H\left(q_{i}, L_{q_{i}}\right)_{i=1, \ldots, m}=\sum_{i=1}^{m} \frac{\partial L}{\partial \dot{q}} \dot{q}-L(q, \dot{q})$ and $p_{i}=\frac{\partial L}{\partial \dot{q}_{i}}, i=1, \ldots, m$, then the above system is equivalent to the Hamiltonian system

$$
\left\{\begin{array}{l}
\dot{q}_{i}=\frac{\partial H}{\partial p_{i}} \\
\dot{p}_{i}=-\frac{\partial H}{\partial q_{i}}+u_{i}, \quad i=1, \ldots, m . \\
y_{i}=q_{i}
\end{array}\right.
$$

The observability distribution of this system will have a constant dimension of $2 m$ so the Hamiltonian system (27) above is locally minimal i.e. it is controllable and observable [10]

\section{Example}

Consider the one-dimensional harmonic oscillator. The equation of motion is given by

$$
\ddot{y}+k^{2} y=0 .
$$

The solution of the inverse problem is given by

$$
L(y, \dot{y})=\frac{1}{2} m \dot{y}^{2}-\frac{1}{2} k y^{2} .
$$

The Hamiltonian is given by

$$
H=\frac{1}{2} \frac{p^{2}}{m}+\frac{1}{2} k y^{2} .
$$

The Hamiltonian system is given by

$$
\left\{\begin{array}{l}
\dot{y}=\frac{p}{m} \\
\dot{p}=-k y
\end{array}\right.
$$

\section{Conclusions}

In this paper it was shown how the Hamiltonian realization problem is described with a view of the inverse problem in classical mechanics being its special case; that is if the inverse problem can be solved, then there is a Hamiltonian realization problem that is solved.

\section{References}

[1] A. J. Van der Schaft, "Controllability and observability or affine nonlinear nonlinear Hamiltonian systems", IEEE Trans, Automomatic Control, Vol AC-27, pp. 490-492, 1992.

[2] W. M. Tulczyjew, "The Legendre transformation, Annales de I'Institut Henri Poincare-Section A - Vol XXII no. 1, pp 102-114, 1977.

[3] S. Bernet and R. G. Cameroon, "Introduction to mathematical control systems", Oxford University Press, New York, 1985.

[4] J. C. Willems and J. C Van der Shaft, "Modelling of dynamical systems using external and internal variables with applications to Hamiltonian systems, Dynamical systems and Microphysics, pp 233-263, Academic Press, New York, 1982.

[5] R. A. Abraham, J. E., Marsden, T. Ratiu, "Manifolds, Tensor analys and Applications, $2^{\text {nd }}$ edition, Springer-Verlag, New York, 1988.

[6] S. P. Banks, "Mathematical theories of Nonlinear systems", Prencice Hall, New York, 1988.

[7] R. M. Santili, Foundations of Theoretical Mechanics I, Springer-Verlag, New York Inc., 1978.

[8] A. J. Van der Schaft, System theoretic description of physical systems, Doctoral Thesis, Mathematical Centrum, Amsterdam, 1984.

[9] W. M. Tulczyjew, Lagrangian submanifolds, statics and dynamics of mechanical systems, Dynamical systems and Microphysics, pp 3-25, Academic Press, New York, 1982.

[10] A. J. Van der Schaft, Controllability and observability for affine nonlinear Hamiltonian systems, IEEE Trans, Automatic Control, Vol AC-27, pp 490-492, 1982. 